\title{
Coherency Evaluation of Spatiotemporal Neural Activities in the Molluscan Olfactory Center Applying Extracellular Recording with Wavelet Analysis
}

\author{
Yuuta Hamasaki ${ }^{1}$, Tomoya Shimokawa ${ }^{1}$, Kohei Ishida ${ }^{1}$, Yoshimasa Komatsuzaki ${ }^{2}$, \\ Satoshi Watanabe ${ }^{3}$, Minoru Saito ${ }^{1 *}$ \\ ${ }^{1}$ Department of Correlative Study in Physics and Chemistry, Graduate School of Integrated Basic Sciences, \\ Nihon University, Tokyo, Japan \\ ${ }^{2}$ Department of Physics, College of Science and Technology, Nihon University, Tokyo, Japan \\ ${ }^{3}$ Center for Disease Biology and Integrative Medicine, Graduate School of Medicine, \\ The University of Tokyo, Tokyo, Japan \\ Email: *msaito@chs.nihon-u.ac.jp
}

Received August 2, 2013; revised September 9, 2013; accepted October 5, 2013

Copyright (C) 2013 Yuuta Hamasaki et al. This is an open access article distributed under the Creative Commons Attribution License, which permits unrestricted use, distribution, and reproduction in any medium, provided the original work is properly cited.

\begin{abstract}
The procerebrum (PC) of the land slug Limax is the olfactory center involved in olfactory discrimination and learning. In the PC, an oscillation of local field potential (LFP) with $0.5-1 \mathrm{~Hz}$ is observed by electrophysiological extracellular recording. Additionally, spatiotemporal neural activities in the PC have been examined using optical recordings. However, extracellular recording is preferable to measure neural activities for a long time with a high speed, while it is not abundant in spatial information. In this study, we therefore attempted to elicit spatial information from extracellular recording. For this purpose, we evaluated spatial information included in the LFP compared with the spatiotemporal neural activities measured by the fluorescent voltage imaging. As a result, aversive odors induced the coherent spatiotemporal neural activities in the PC, and the increase in coherency was observed as a change in the LFP waveform. It was also evaluated as a decrease in entropy by analyzing the LFP oscillation patterns and wavelet analysis. Thus, although the LFP provides only one series of signals, the coherency of the spatiotemporal neural activities in the PC can be evaluated by applying extracellular recording with wavelet analysis.
\end{abstract}

Keywords: Land Slug; Extracellular Recording; Fluorescent Voltage Imaging; Olfactory Center; Spatiotemporal Neural Activity; Coherency; Wavelet Analysis

\section{Introduction}

The procerebrum (PC) of the land slug Limax is the olfactory center involved in olfactory discrimination and learning $[1,2]$. In the PC, an oscillation of local field potential (LFP) with $0.5-1 \mathrm{~Hz}$ is observed by electrophysiological extracellular recording [3]. The oscillatory activity is supposed to arise from synaptic interactions of two types of interneurons [4-6]. Additionally, previous studies using optical recordings revealed spatiotemporal activity patterns in the PC $[4,7,8]$. The oscillation has a phase delay along the distal-proximal axis, while the neural activities along the anterior-posterior axis are synchronized, resulting in the propagation of waves from distal to proximal regions.

${ }^{*}$ Corresponding author.
Optical recordings provide direct measurement for spatiotemporal neural activities. Optical recordings, however, have some defects. For example, in the fluorescent voltage imaging, voltage sensitive dyes generally exhibit a relatively small change in the fluorescence intensities, resulting in a low $\mathrm{S} / \mathrm{N}$ ratio, and often exhibit photobleaching and phototoxity. Therefore, there are not many reports on long time stable measurement by the fluorescent voltage imaging. Additionally, high speed measurement is difficult for imaging. Recently, we have developed an imaging system which enables us to measure spatiotemporal neural activities for a long time with a high speed and a high $\mathrm{S} / \mathrm{N}$ ratio [9]. However, extracellular recording is still preferable for long time and high speed measurement, while it is not abundant in spatial information. It is therefore important to elicit spatial in- 
formation from extracellular recording.

In this study, we first measured the LFP oscillations in the PC of Limax valentianus by the conventional extracellular recording. We next evaluated spatial information included in the present LFP compared with the spatiotemporal neural activities measured by the fluorescent voltage imaging. Finally, we analyzed the LFP oscillations by the wavelet analysis to evaluate the coherency of the spatiotemporal neural activities in the PC. The wavelet analysis is a frequency analysis, which is more suitable for physiological data than Fourier-based analyses.

\section{Materials and Methods}

\subsection{Preparations}

The land slug Limax valentianus was anesthetized by injection of $35 \mathrm{mM} \mathrm{Mg}^{2+}$ solution into the body cavity, and the central nervous system (CNS) was isolated with tentacles from the body in the dissection solution (composition in $\mathrm{mM}: \mathrm{NaCl} 35, \mathrm{KCl} 2, \mathrm{MgCl}_{2} 28, \mathrm{CaCl}_{2} 4.9$, D-glucose 5, HEPES-NaOH 5 (pH 7.6)). The PC is located at the distal part of each cerebral ganglion.

\subsection{Extracellular Recording}

The recording chamber was consisted of two compartments with a slit between them. The isolated CNS was placed into one of the compartments filled with the slug saline (composition in $\mathrm{mM}: \mathrm{NaCl} 70, \mathrm{KCl} 2, \mathrm{MgCl}_{2} 4.7$, $\mathrm{CaCl}_{2}$ 4.9, D-glucose 5, HEPES-NaOH 5 (pH 7.6)), and tentacles were placed to pass through the slit to the other compartment exposed air. The slit was sealed with silicon grease, and the odor stimulus was applied to the tentacles by a continuous flow from the odor source. The LFP was measured through a glass suction electrode (tip diameter $=80 \mu \mathrm{m}$ ) filled with the slug saline. The reference electrode was placed into the slug saline in the former compartment. The signals were amplified with an extracellular recording amplifier (ER-1, Cygnus, USA) and transferred through an AD converter (PowerLab, ADInstruments, Australia) into a personal computer (Dell Precision T5600, Dell, USA) with $1 \mathrm{kHz}$ sampling frequency. The sampled signals are expressed as $V(\mathrm{t})=$ $\left\{V_{1}, V_{2}, \cdots, V_{\mathrm{M}}\right\}$.

\subsection{Fluorescent Voltage Imaging}

For the fluorescence measurement, a voltage sensitive dye Di-4-ANEPPS (Wako, Japan) was used. The isolated CNS was loaded for $30 \mathrm{~min}$ at room temperature with 86 $\mu \mathrm{M}$ Di-4-ANEPPS in the presence of $0.06 \%$ cremophor EL and $0.6 \%$ ethanol in the slug saline. After the dye loading, the preparation was washed with the slug saline.

The stained preparation was placed on the recording chamber mentioned above. The chamber was then mounted on the stage of a microscope (E-FN1, Nikon, Japan) with a $10 \times$ objective lens (Plan Apo, 0.45 NA, Nikon, Japan). The dye was excited by a LED of $530 \mathrm{~nm}$ with a half width of $25 \mathrm{~nm}$ (LEX2-G, Brain Vision, Japan) through an excitation filter (EX510-560). The emitted fluorescence of $705 \mathrm{~nm}$ was detected through a dichroic mirror (DM575) and a barrier filter (BA590). The fluorescence images (1024 pixel $\times 1024$ pixel) were acquired at a rate of $10 \mathrm{~ms} /$ flame by a sCMOS camera (Zyla, Andor, Ireland). The image sequences were stored into a personal computer (Dell Precision T5600, Dell, USA).

\subsection{Wavelet Analysis}

The wavelet analysis is a frequency analysis, which is different from Fourier-based analyses in which time stationarity is assumed for the signals. Physiological signals are non-stationary, for example, the present LFP oscillation pattern is transiently changed by odor stimuli. Because an assumption on time stationarity is not needed for the wavelet analysis, it has been applied to physiological signal analyses [10-13]. In the present study, we also used a similar method to the previous studies as briefly described below.

The wavelet analysis is based on the introduction of an appropriate orthogonal basis, which is an orthonormal basis when normalized, and the signals are characterized based on the distribution of amplitude within basis. The orthonormal basis is called mother wavelet. We employed the "Daubechies" function as mother wavelet $\psi$ [14]. The wavelet expansion is expressed as

$$
\begin{aligned}
& V(t)=\sum_{j=1}^{N} \sum_{k} C(j, k) \psi_{j, k}(t), \\
& C(j, k)=\int_{R} V(t) \psi_{j, k}(t) \mathrm{d} t, \\
& \psi_{j, k}(t)=\frac{1}{\sqrt{2^{j}}} \psi\left(2^{-j} t-k\right) .
\end{aligned}
$$

Here, $C(j, k)$ are wavelet coefficients. $j$ is a resolution level and $k$ is a time index. $N$ is the number of the resolution levels, which was set to 12 in this study. The resolution level $j$ determines one frequency band. The concept of energy is linked with that in the Fourier theory. The mean wavelet energy at frequency band $j$ in time window $i$ is expressed as

$$
E_{j}^{(i)}=\frac{1}{N_{j}} \sum_{k^{\prime}}\left|C\left(j, k^{\prime}\right)\right|^{2} .
$$

The corresponding squared coefficients, $\left|C\left(j, k^{\prime}\right)\right|^{2}$, are summed over time index $k^{\prime}$ within the time window $i . N_{j}$ is the number of the wavelet coefficients. The relative wavelet energy in the time window $i$ is expressed as

$$
P_{j}^{(i)}=E_{j}^{(i)} / E_{\text {tot }}^{(i)}, E_{\text {tot }}^{(i)}=\sum_{j} E_{j}^{(i)} .
$$


$P_{j}^{(i)}$ represents the probability distribution of the energy, where $\sum_{j=1}^{N} P_{j}^{(i)}=1$. The wavelet entropy in the time window $i$ is expressed as

$$
S^{(i)}=-\sum_{j=1}^{N} P_{j}^{(i)} \ln P_{j}^{(i)} .
$$

\section{Results and Discussion}

Figure 1 shows time changes in the LFP in the PC. The LFP is induced by the electric current between the glass electrode and the reference. When the electric current is from the glass electrode to the reference, the LFP become upward. As another group reported, the LFP showed an oscillation with $0.5-1 \mathrm{~Hz}$ (Figure 1(a)). While 3 to $5 \mu \mathrm{m}$ tip glass electrodes were used in the previous reports $[3,4,7]$, however, larger-bore ( $80 \mu \mathrm{m}$ tip) glass electrodes were used in the present study. It is therefore supposed that more spatial information is included in the present LFP, which is discussed below. In Figure 1, no change in the LFP oscillation pattern was seen in a wet air stimulus (Figure 1(a)), while a change was seen in an aversive odor (isoamyl acetate) stimulus (Figure 1(b)). Although the frequency estimated from peak-to-peak intervals was not much changed by the odor stimulus, the waveform became different as shown in Figure 2. The waveform had shoulders (thin line in Figure 2(a)) and sometimes some peaks (shown later) before the odor stimulus. The characteristics in the waveform are supposed to be due to a phase difference among neural activities in the measurement area of the glass electrode.

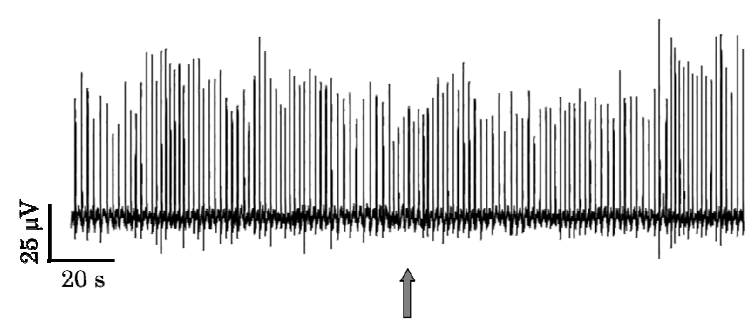

(a)

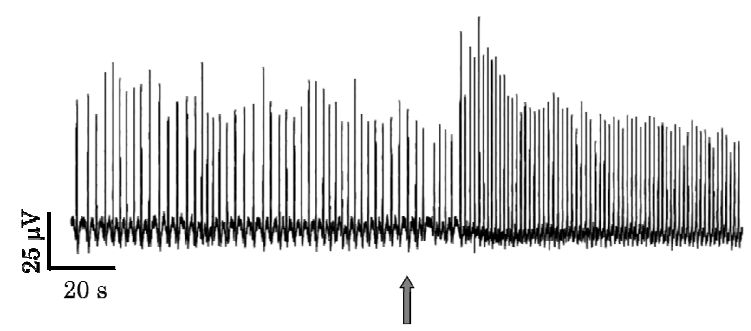

(b)

Figure 1. LFP oscillations in the PC. (a) Control (a wet air stimulus); (b) An aversive odor (isoamyl acetate) stimulus. The wet air or odor stimulus was applied at the time shown by the gray arrow.
After the odor stimulus, however, it had one peak and a sharp shape (thick line in Figure 2(a)), which suggests a decrease in the phase difference among the neural activities. Similar results were obtained for another aversive odor (cineol). The half width of the waveform was decreased by the aversive odor stimuli (Figure 2(b)). Thus, the present LFP measurement suggests that the aversive odors induce spatially coherent activities in the PC. To confirm it, we next examined the spatiotemporal neural activities in the PC using the fluorescent voltage imaging.

Figure 3 shows time changes in the fluorescence intensity of the dye (Di-4-ANEPPS) in the PC. The four ROIs with 64 pixel $\times 64$ pixel (about $40 \mu \mathrm{m} \times 40 \mu \mathrm{m}$ ) were put on the fluorescent images along the distalproximal axis of the PC (Figures 3(a) and (b)). The changes in the fluorescence intensity $(\Delta F)$ are expressed as relative values to the basal level with $\%$. The fluorescence intensity of each ROI showed an oscillation corresponding to the LFP oscillation simultaneously measured (Figures 3(c)). Here, the fluorescence increase of the dye $(\Delta F>0)$ corresponds to hyperpolarization of neurons. The hyperpolarization induces the electric currents to surroundings. The summation of the electric currents

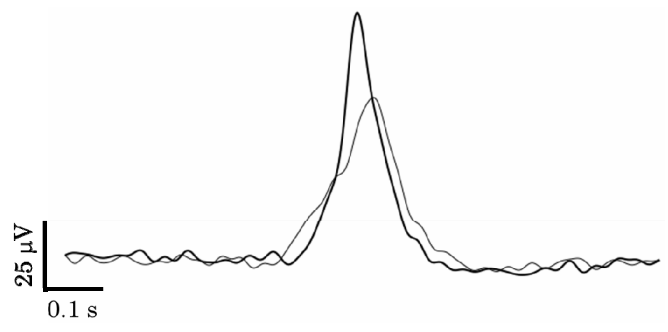

(a)

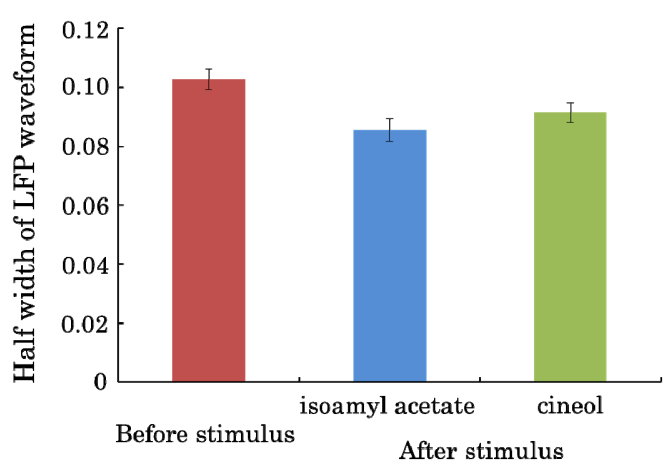

(b)

Figure 2. (a) Comparison of the LFP waveform after the aversive odor (isoamyl acetate) stimulus (thick line) to that before the odor stimulus (thin line); (b) Half widths of the LFP waveform. They are averaged for the waveforms within 5 - $10 \mathrm{~s}$ before or after the aversive odor (isoamyl acetate or cineol) stimulus in some trials. The data are expressed as means \pm SEM. $n=119$ before the odor stimulus, $n=80$ after the isoamyl acetate stimulus and $n=40$ after the cineol stimulus. 


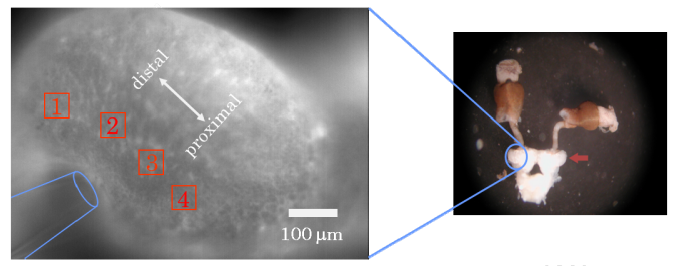

(a)

(b)
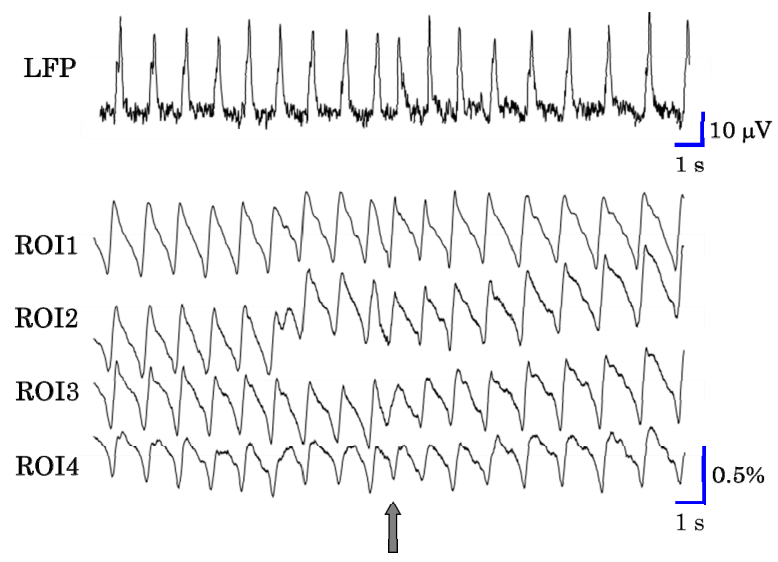

(c)

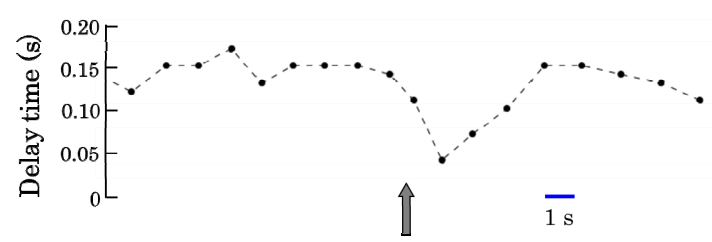

(d)

Figure 3. (a) Fluorescence image of the PC. The ROI flames for fluorescence analysis (red squares) and the glass electrode (blue figure) for extracellular recording are shown; (b) Preparation of the central nervous system with tentacles. The blue circle and the red arrow show the left and right PCs, respectively; (c) Time changes in the LFP (upper figure) and the fluorescence intensity in the ROI flames (lower figure). The LFP was simultaneously measured by extracellular recording; (d) Delay times estimated from the peaks of fluorescence oscillations in the flame 1 and the flame 4 . The aversive odor (isoamyl acetate) stimulus was applied at the time shown by the gray arrow in (c) and (d).

induces the LFP. When the total electric current is from the glass electrode to the reference, the LFP becomes upward.

As shown in Figure 3(c), the fluorescence oscillation had a phase delay from distal to proximal region in the PC before the odor stimulus, and the delay time was 100 - $150 \mathrm{~ms}$ between distal and proximal regions (Figure 3(d)). Here, the delay time was estimated by the downward peaks, because they were more easily detected than the upward peaks. On the other hand, the phase delay disappeared after the aversive (isoamyl acetate) stimulus (Figure 3(d)). Figure 4 shows pseudo-color images obtained from $\Delta F / \Delta t$ in each image element $(16$ pixel $\times 16$ pixel (about $10 \mu \mathrm{m} \times 10 \mu \mathrm{m})$ ). $\Delta F / \Delta t$ in each image element corresponds to each electric current to surroundings. This figure also shows that the neural activities propagate from distal to proximal region of the PC before the odor stimulus and they became coherent all over the PC after the odor stimulus. As mentioned above, these results were already supposed from the change in the LFP waveform. Therefore, it is suggested that the coherency evaluation of the spatiotemporal neural activities in the PC is possible from the present LFP measurement.

We next evaluated the spatial information included in the present LFP by comparing with the fluorescent voltage imaging. Figure 5 shows comparisons of the waveforms between the LFP (blue line) and the summation of $\Delta F / \Delta t$ (red line) in the image elements. Here, the waveforms were obtained before the odor stimulus. One of the LFP waveforms had two peaks (see the left LFP waveform). To obtain the waveforms of the summation of $\Delta F / \Delta t$, a line of 25 image elements was put perpendicularly to the distal-proximal axis on the center of the glass electrode, and then the number of lines was gradually increased along the distal-proximal axis (see the area shown with the red square in the fluorescence image). When the number of lines was 3, that is, the width of the area was about $30 \mu \mathrm{m}$, the summation of $\Delta F / \Delta t$ could not reproduce two peaks in the LFP (see the part shown with the gray arrow in Figure 5(a)). On the other hand, when the number of lines was 44 , that is, the width of the area was about $440 \mu \mathrm{m}$, the summation of $\Delta F / \Delta t$ became too wide compared with the LFP waveform (see the part shown with the gray arrow in Figure 5(c)). As seen from Figure 5(b), when the number of lines was 32, that is, the width of the area was about $320 \mu \mathrm{m}$, the summation of $\Delta F / \Delta t$ could well reproduce the LFP waveform. We therefore conclude that the present LFP includes spatial information within the area of $300-350 \mu \mathrm{m}$ width along the distal-proximal axis.

Finally, we attempted to evaluate the coherency of the spatiotemporal neural activities in the PC from the LFP oscillation patterns by the wavelet analysis. Figure 6 shows time changes in the wavelet energy in each frequency band and the wavelet entropy. In this figure, the wavelet energy is a relative value to the total energy, which is the relative wavelet energy $P_{j}^{(i)}$ in Equation (1) (Figure 6(a)), and the wavelet entropy is also a relative value obtained by normalizing $S^{(i)}$ in Equation (2) by the maximum entropy $S_{\max }$ (Figure 6(b)). $S_{\max }$ is obtained when the energy is distributed equally to each frequency band, that is, $S_{\max }=\ln 12$ in this study. In wavelet analysis, the resolution level $j$ determines one frequency band. After the aversive (isoamyl acetate) stimulus, the energy in the $0.45-0.90 \mathrm{~Hz}$ band increased and that in the 2.40 $5.00 \mathrm{~Hz}$ band decreased, and the entropy also decreased. Similar results were obtained for the other aversive 

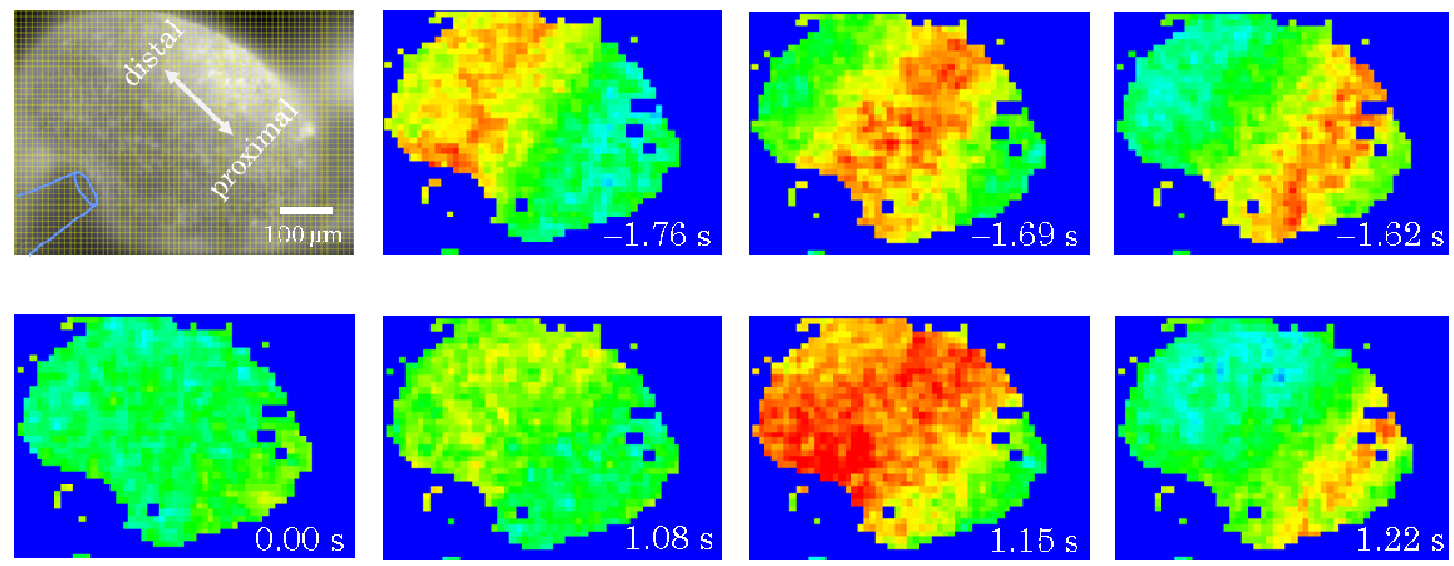

Figure 4. Pseudo-color images of the spatiotemporal neural activities in the PC. The upper left figure shows the fluorescence image of the PC. The image elements are shown with the yellow mesh in the fluorescence image. The glass electrode for extracellular recording (blue figure) is also shown. The pseudo-color images were obtained from $\Delta F / \Delta t$ in each image element (16 pixel $\times 16$ pixel (about $10 \mu \mathrm{m} \times 10 \mu \mathrm{m})$ ). The times before and after the aversive odor (isoamyl acetate) stimulus are shown. The odor stimulus was applied at $0.00 \mathrm{~s}$.
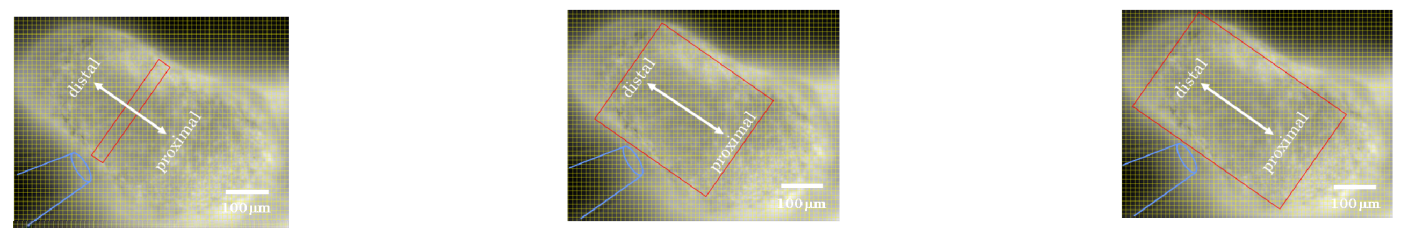

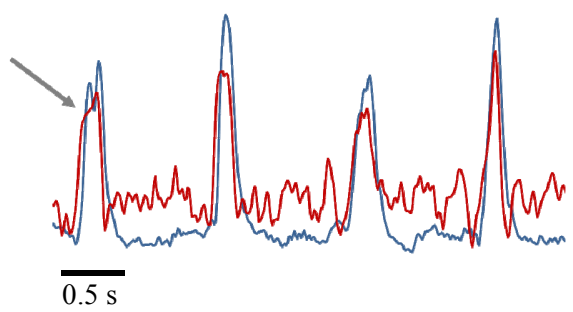

(a)

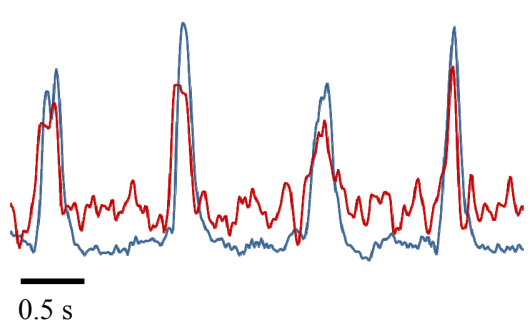

(b)

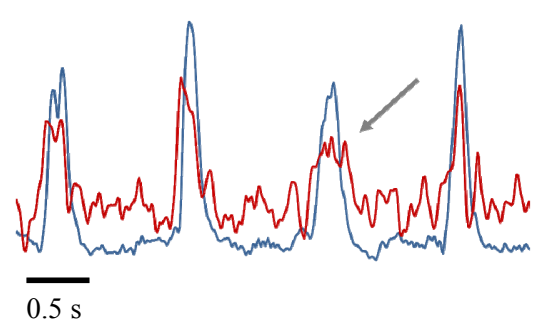

(c)

Figure 5. Comparisons of the waveform between the LFP (blue line) and the summation of $\Delta F / \Delta t$ (red line) in the image elements. The upper figure in each figure shows the fluorescence image of the PC. The image elements are shown with the yellow mesh. A line of 25 image elements was put perpendicularly to the distal-proximal axis on the center of the glass electrode which is shown as the blue figure. The number of lines was gradually increased along the distal-proximal axis. The area shown with the red square in the fluorescence image was used to estimate the summation of $\Delta F / \Delta t$ in the image elements: (a) 3 lines; (b) 32 lines; (c) 44 lines. The gray arrows in (a) and (c) are explained in the text.

(cineol) stimulus. Figure 7 shows the wavelet energy in each frequency band and the wavelet entropy which were averaged within 15 time windows (about $60 \mathrm{~s}$ ) before or after the aversive odor (isoamyl acetate or cineol) stimulus in some trials. The energy was widely distributed, especially in three frequency bands $(0.45-0.90 \mathrm{~Hz}, 0.90$ - $2.40 \mathrm{~Hz}, 2.40-5.00 \mathrm{~Hz}$ ), before the odor stimulus. After that, however, it was concentrated in the $0.45-0.90$ $\mathrm{Hz}$ and $0.90-2.40 \mathrm{~Hz}$ bands (Figure 7(a)). This result is attributed to the decrease in the width of the waveform (see Figure 2(a)), because the frequency estimated from peak-to-peak intervals was not much changed. Based on the change in the frequency distribution, the wavelet entropy decreased after the aversive odor stimuli (Figure 7(b)). As mentioned above, the aversive odors induced the coherent spatiotemporal neural activities in the PC. The present results therefore show that the coherency of the spatiotemporal neural activities can be evaluated from the LFP oscillation patterns by the wavelet entropy.

As well known, entropy is a measure of the degree of order/disorder of the system [15]. A decrease in entropy shows an increase in coherency of the system, resulting in acquisition of information. In the present study, therefore, the decrease in the wavelet entropy, which results from the coherent spatiotemporal neural activities in the PC, might lead to acquisition of odor information in the land slug. 


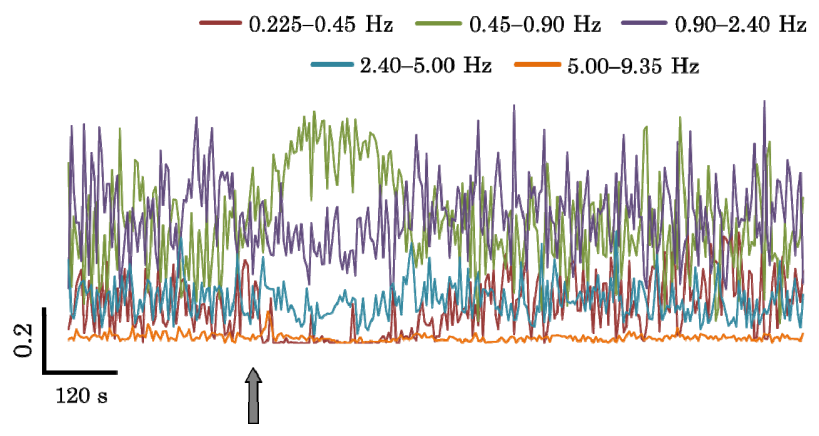

(a)

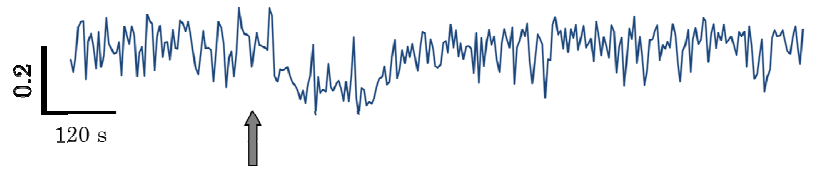

(b)

Figure 6. (a) Time change in the wavelet energy in each frequency band; (b) Time change in the wavelet entropy. The aversive odor (isoamyl acetate) stimulus was applied at the time shown by each gray arrow.

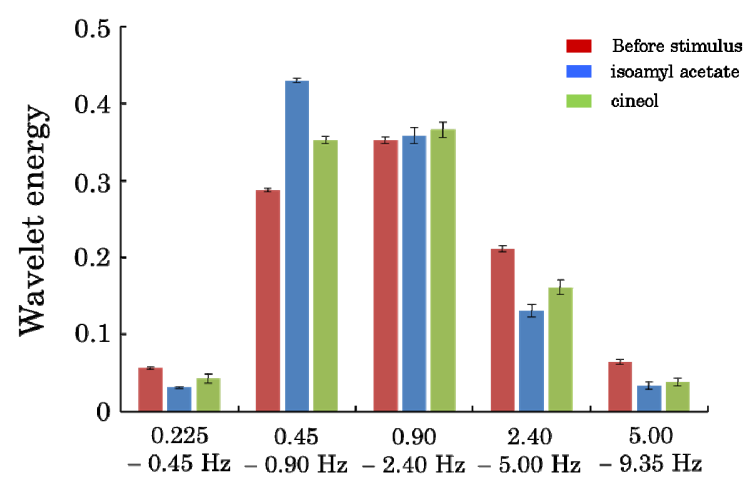

(a)

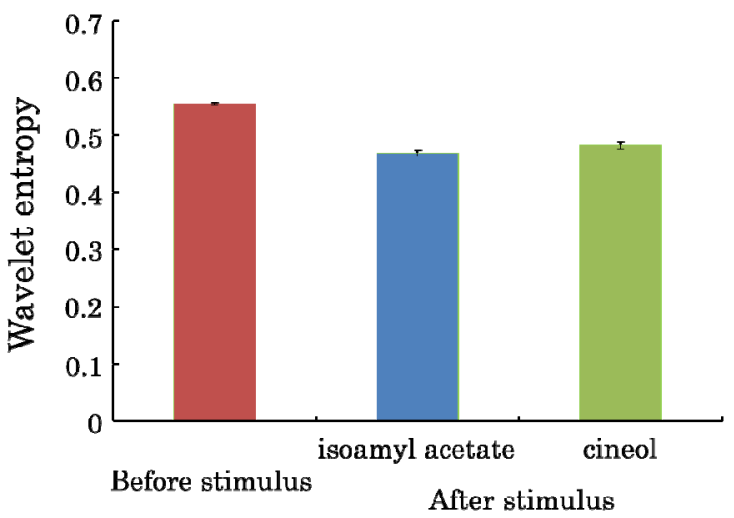

(b)

Figure 7. (a) Wavelet energy in each frequency band; (b) Wavelet entropy. They are averaged within 15 time windows (about $60 \mathrm{~s}$ ) before or after the aversive odor (isoamyl acetate or cineol) stimulus in some trials. The data are expressed as means \pm SEM. $n=735$ before the odor stimulus, $n=255$ after the isoamyl acetate stimulus and $n=240$ after the cineol stimulus.

\section{Conclusion}

In the present study, we first compared the LFP oscillation measured by extracellular recording with the spatiotemporal neural activities measured by the fluorescent voltage imaging. As a result, the aversive odors induced the coherent spatiotemporal neural activities in the PC, and the increase in coherency was observed as a change in the LFP waveform, that is, a decrease in the width of the waveform. Thus, spatial information was included in the present LFP. We next evaluated the coherency change by analyzing the LFP oscillation patterns by wavelet analysis. The result showed that the wavelet energy became concentrated in two frequency bands and the wavelet entropy was decreased by the aversive odor stimuli. In conclusion, although the LFP provides only one series of signals, the coherency of the spatiotemporal neural activities in the PC can be evaluated by applying extracellular recording with wavelet analysis.

\section{Acknowledgements}

This study was partly supported by KAKENHI grant (No. 22560348 to M.S.) from Japan Society for the promotion of Science and Nihon University Academic Research grant (Total Research 11-002 and 12-002 to M.S.).

\section{REFERENCES}

[1] T. Kimura, H. Suzuki, E. Kono and T. Sekiguchi, "Mapping of Interneurons That Contribute to Food Aversive Conditioning in the Slug Brain," Learning \& Memory, Vol. 4, No. 5, 1998, pp. 376-388. http://dx.doi.org/10.1101/lm.4.5.376

[2] T. Teyke and A. Gelperin, "Olfactory Oscillation Augment Odor Discrimination Not Odor Identification by $\mathrm{Li}$ max CNS," NeuroReport, Vol. 10, No. 5, 1999, pp. 10611068 .

http://dx.doi.org/10.1097/00001756-199904060-00030

[3] A. Gelperin and D. W. Tank, "Odour-Modulated Collective Network Oscillations of Olfactory Interneurons in a Terrestrial Mollusk," Nature, Vol. 345, No. 6274, 1990, pp. 437-440. http://dx.doi.org/10.1038/345437a0

[4] D. Kleinfeld, K. R. Delaney, M. S. Fee, J. A. Flores, D. W. Tank and A. Gelperin, "Dynamics of Propagating Waves in the Olfactory Network of a Terrestrial Mollusk: An Electrical and Optical Study," Journal of Neurophysiology, Vol. 72, No. 3, 1994, pp. 1402-1419.

[5] S. Watanebe, S. Kawahara and Y. Kirino, "Morphological Characterization of the Bursting and Nonbursting Neurons in the Olfactory Centre of the Terrestrial Slug Limax marginatus," Journal of Experimental Biology, Vol. 201, No. 7, 1998, pp. 925-930.

[6] S. Watanabe and Y. Kirino, "Selective Calcium Imaging of Olfactory Interneurons in a Land Mollusk," Neuroscience Letters, Vol. 417, No. 3, 2007, pp. 246-249. http://dx.doi.org/10.1016/j.neulet.2007.02.083

[7] K. R. Delaney, A. Gelperin, M. S. Fee, J. A. Flores, R. 
Gervais, D. W. Tank and D. Kleinfeld, "Waves and Stimulus-Modulated Dynamics in an Oscillating Olfactory Network," Proceedings of National Academy of Sciences of USA, Vol. 91, No. 2, 1994, pp. 669-673. http://dx.doi.org/10.1073/pnas.91.2.669

[8] S. Inoue, S. Kawahara, S. Toda, S. Watanabe and Y. Kirino, "Selective Optical Recording of the Neural Activity in the Olfactory Center of the Land Slug Using a Calcium Indicator Dye," Bioimages, Vol. 6, No. 2, 1998, pp. 5967.

[9] Y. Hamasaki, M. Hosoi, S. Nakada, T. Shimokawa and M. Saito, "Fluorescent Voltage Imaging Technique for the Measurement of Molluscan Neural Activities," Open Journal of Biophysics, Vol. 3, No. 1A, 2013, pp. 54-58. http://dx.doi.org/10.4236/ojbiphy.2013.31A007

[10] R. Q. Quiroga, O. A. Rosso, E. Başar and M. Schürmann, "Wavelet Entropy in Event-Related Potentials: A New Method Shows Ordering of EEG Oscillations," Biological Cybernetics, Vol. 84, No. 4, 2001, pp. 291-299. http://dx.doi.org/10.1007/s004220000212

[11] O. A. Rosso, S. Blanco, J. Yordanova, V. Kolev, A. Figlioa, M. Schürmann and E. Başar, "Wavelet Entropy: A New Tool for Analysis of Short Duration Electrical Sig- nals," Journal of Neuroscience Methods, Vol. 105, No. 1, 2001, pp. 65-75.

http://dx.doi.org/10.1016/S0165-0270(00)00356-3

[12] A. Schütt, O. A. Rosso and A. Figliola, "A Discovery of New Features of Gastropod Local Field Potentials by Application of Wavelet Tools," Journal of Neuroscience Methods, Vol. 119, No. 1, 2002, pp. 89-104. http://dx.doi.org/10.1016/S0165-0270(02)00184-X

[13] A. Schütt, I. Ito, O. A. Rosso and A. Figliola, "Wavelet Analysis Can Sensitively Describe Dynamics of Ethanol Evoked Local Field Potentials of the Slug (Limax marginatus) Brain," Journal of Neuroscience Methods, Vol. 129, No. 2, 2003, pp. 135-150.

http://dx.doi.org/10.1016/S0165-0270(03)00200-0

[14] I. Daubechies, "Orthonormal Bases of Compactly Supported Wavelets," Communications on Pure and Applied Mathematics, Vol. 41, No. 7, 1988, pp. 909-996. http://dx.doi.org/10.1002/cpa.3160410705

[15] C. E. Shannon, "A Mathematical Theory of Communication Bell System," Bell System Technical Journal, Vol. 27, No. 3, 1948, pp. 379-423; Vol. 27, No. 4, 1948, pp. 623656. 